\title{
Advanced Therapy Medicinal Products Challenges and Perspectives in Regenerative Medicine
}

\author{
Aspasia Goula ${ }^{\mathrm{a}}$, Vasiliki Gkioka ${ }^{\mathrm{b}, \mathrm{d}}$, Efstathios Michalopoulos ${ }^{\mathrm{b}}$, Michalis Katsimpoulas ${ }^{\mathrm{b}}$, \\ Michel Noutsias $^{\mathrm{c}}$, Eirini Faidra Sarri ${ }^{\mathrm{b}}$, Catherine Stavropoulos ${ }^{\mathrm{b}}$, Alkiviadis Kostakis ${ }^{\mathrm{b}}$
}

\begin{abstract}
Recently, the design and development of a modern health policy in the field of regenerative medicine leads to the formation of a new and integrated cognitive field, which requires systematic research and study in order to produce innovative answers and best practices. Advanced therapy medicinal products (ATMPs) is a new product category, which is at the heart of concern since it has to deal with diseases in which traditional medicine has proven to be ineffective so far. The aim of this review is to provide evidence for the state of the art ATMPs and their modern applications in the field of regenerative medicine. The ATMPs are characterized by a great heterogeneity and variation in methods of isolation, which cover the entire spectrum from a single intravenous injection to a surgical placement. Clinical development of ATMP encounters specific challenges due to the nature of the product and the limited availability of non-clinical data. The gold standard of a controlled, randomized, clinical trial may not be feasible or ethically justified for all indications, particularly in life-threatening diseases, where there is no satisfactory standard of care. Therefore, the European Commission (EC) took initiatives in order to set standards and operating rules concerning authorization and supervision of ATMPs and on pharmacovigilance in relation to them. The European Union (EU) Regulation 1394/2007 provides the possibility of exceptions. In particular, the "hospital exemption" allows for the administration of an ATMP without a license on certain conditions. Although the Regulation 1394/2007 has led to the commercial exploitation of ATMPs, the reality today, 11 years after its first implementation, is completely different. While the Committee for Advanced Therapies (CAT) has already registered 285 products as
\end{abstract}

Manuscript submitted August 26, 2020, accepted September 19, 2020

Published online December 18, 2020

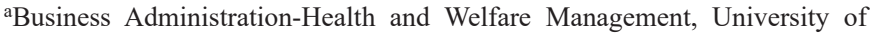
West Attica, Egaleo, Greece

bBiomedical Research Foundation Academy of Athens, 4th Soranou Efessiou Str., 11527 Athens, Greece

${ }^{\mathrm{c}}$ Mid-German Heart Center, Department of Internal Medicine III, Division of Cardiology, Angiology and Intensive Medical Care, University Hospital Halle, Martin-Luther-University Halle-Wittenberg, Ernst-Grube-Strasse 40, D-06120 Halle (Saale), Germany

${ }^{\mathrm{d} C}$ Corresponding Author: Vasiliki Gkioka, Hellenic Cord Blood Bank, Biomedical Research Foundation, Academy of Athens (BRFAA), Athina 11527, Greece. Email: vgkioka@gmail.com

doi: https://doi.org/10.14740/jocmr3964
ATMPs, only 10 licenses were granted which only remained six (the rest related to products withdrawn). The key players in the development and delivery of ATMPs still remain the academic/research centers and small and medium-sized enterprises; while the involvement of pharmaceutical companies is focusing on recent developments in the treatment of oncological incidents with in vitro modified cytotoxic $\mathrm{T}$ lymphocytes, and chimeric antigen receptor (CAR)-T cells.

Keywords: Advanced therapy medicinal products; Randomize clinical trial; EU regulation; CAT; Quality and safety standards

\section{Introduction}

Nowadays, health policies are directly affected by the type, extent and complexity of diseases affecting the population, and at the same time require the creation of a health care system that fulfils health needs and promotes good health. This system must be based on scientific criteria and principles and shall take into account progress in medicine, biology, genetics, biotechnology, economics, as well as the needs of citizens to protect and promote their health [1].

Recently, the design and development of a modern health policy in the field of regenerative medicine leads to the formation of a new and integrated cognitive field that requires systematic research and study in order to produce innovative answers and best practices. It also requires systematic investigation and the verification of cases of a complete analysis of the health system, in order to continuously improve the knowledge of human resources of the health services, as well as to enhance the staff with specific skills and competences required by the developments in the field of regenerative medicine [1].

The term "regenerative medicine" refers to methods to replace or regenerate human cells, tissues or organs in order to restore or establish normal function. This includes cell therapies, tissue engineering, gene therapy and biomedical engineering techniques, as well as more traditional treatments involving pharmaceuticals, biologics and devices.

As mentioned, conventional drugs usually contain chemicals or proteins as active substances. However, recent advances in science and technology have led to the emergence of medicinal products consisting of genes or cells. While these products can also be subsumed under the umbrella term "biological drugs" they have distinct and unique characteristics 
Table 1. Classification, Definition and Applications of ATMPs

\begin{tabular}{|c|c|c|}
\hline Classification & Definition & Applications \\
\hline $\begin{array}{l}\text { Gene-based therapeutic } \\
\text { medicinal products }\end{array}$ & $\begin{array}{l}\text { Gene transfer-based approaches which are leading } \\
\text { to therapeutic, prophylactic or diagnostic effect. }\end{array}$ & $\begin{array}{l}\text { Inherited disease } \\
\text { Cancer therapy } \\
\text { Tissue regeneration (e.g., lost of sight) }\end{array}$ \\
\hline $\begin{array}{l}\text { Somatic cell therapeutic } \\
\text { medicinal products }\end{array}$ & $\begin{array}{l}\text { Cell-based approaches which are including } \\
\text { in vitro manipulating cells or tissues with } \\
\text { therapeutic, prophylactic or diagnostic effect. }\end{array}$ & $\begin{array}{l}\text { Products against immune disease } \\
\text { Parkinson's disease, ALS, Alzheimer's disease } \\
\text { Cartilage defect } \\
\text { Product for cardiac repair } \\
\text { Skin replacement } \\
\text { Cancer immunotherapy }\end{array}$ \\
\hline $\begin{array}{l}\text { Tissue-engineered } \\
\text { medicinal products }\end{array}$ & $\begin{array}{l}\text { Tissue engineered-based approaches that } \\
\text { can be applied to repair, regenerate or } \\
\text { replace human tissues or organs. }\end{array}$ & $\begin{array}{l}\text { Small diameter vascular grafts } \\
\text { Trachea replacement } \\
\text { Tissue-engineered esophagus } \\
\text { Liver and kidney implantation } \\
\text { Nerve conduits }\end{array}$ \\
\hline
\end{tabular}

ATMPs: advanced therapy medicinal products; ALS: amyotrophic lateral sclerosis.

that warranted their classification as a distinct type of drugs; these specialized medicinal products are therefore referred to as advanced therapy medicinal products (ATMPs).

ATMP is a new product category, which is at the heart of concern since it has to deal with diseases in which traditional medicine has proven to be ineffective so far. The main aim of this review article is to provide comprehensive information of ATMPs, which could be applied in various regenerative medicine and tissue engineering applications. Moreover, ATMPs could be a valuable tool for the physicians, for the proper administration of life-threatening diseases, thus could be applied in personalized regenerative medicine.

\section{Categories and Characteristics of ATMPs}

The ATMPs consist of three basic categories of products: 1) gene therapy (gene therapy medicinal products (GTMPs)); 2) somatic cell therapies (as defined in part IV of Annex I to Directive 2001/83/EC) [2]; and 3) tissue engineering products, as well as any combination of the above. These are products that use human cells and tissues which have undergone gene or other treatment or modification in the laboratory and are not fully covered by the Directive 2004/23/EC [3]. The donor and the recipient of the cells/tissues may be the same individual (autologous use) or different (allogeneic use) (Table 1).

Somatic cell therapy products pertain to cell-based medicinal products (CBMPs) treatments. These products are extremely heterogeneous due to their origin, original harvested material, in vitro handling degree and production process. These characteristics include the type of cell population and its stage of development or differentiation (embryonic or adult stem cells or early progenitor cells, induced pluripotent stem cells (IPSs) or differentiated cells).

Tissue engineered products belong to the CBMPs catego- ry and have been developed to structurally repair various tissue damages (e.g., cornea, cardiac or hepatic tissue, blood vessels, cartilage or bone). These types of cellular therapies are sometimes associated with structural components that promote the formation of a three-dimensional tissue structure which leads to favorable cellular interactions. The active substance of these products may be an immature cell population (e.g., stem cell progenitor cells) or a differentiated stem cell population, which will form the final tissue (e.g., skin) [4].

\section{Fields of Application}

Until today, the main goal of ATMPs is incurable diseases, orphan diseases and chronic diseases that traditional drugs are unable to treat. In particular, genetic and metabolic diseases, degenerative neurological diseases, malignancies and hematological diseases, serious cardiological and orthopedic cases are the field of their application, whereas their action for the regeneration of damaged tissues is essentially responsible for the rapid development of the field of regenerative medicine (Fig. 1) [5].

The development of ATMP is a result of extensive work of academic and research centers without the involvement of pharmaceutical companies, and the early stages of their implementation, given the lack of a comprehensive regulatory framework, were applied in the context of small-scale clinical trials or were administered as a consolation therapy.

Apart from the specificities related to the origin of the raw material (human biological material), their development and availability, the ATMP are characterized by a great heterogeneity. Indeed, so far a large number of cell populations and tissues have been reported with potential pharmaceutical activity. Also, there is a great variation in methods of isolation, culturing and processing/modification of tissues and cells, whereas 


\section{Advanced Therapeutic Medicinal Products}

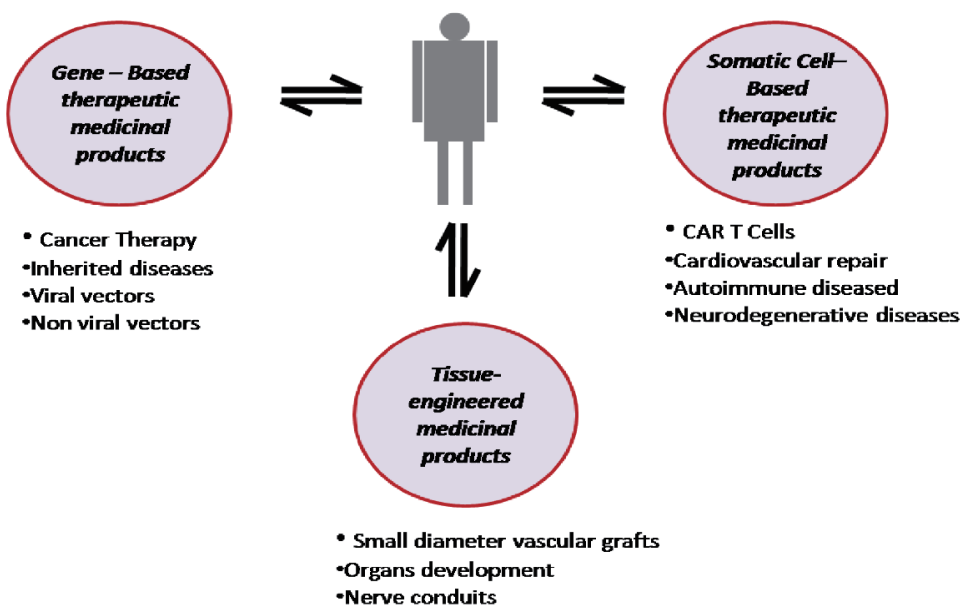

Figure 1. Schematic representation of advanced medicinal therapeutic products. CAR: chimeric antigen receptor.

the methods of administration cover the entire spectrum from a single intravenous injection to a surgical placement.

\section{Clinical Use of Advanced Therapy Products: Modern Challenges}

ATMPs are at the forefront of innovation, offering treatment options for diseases that currently have little or no effective treatment options. Therefore, the interest in the use of ATMP is important, but they have both positive and negative results [6].

Clinical development of ATMP encounters specific challenges due to the nature of the product and the limited availability of non-clinical data. In addition, their mode of administration may be invasive or require special equipment to place them in the human body, a procedure which raises additional quality assurance problems associated with the administration process (Table 2).

The gold standard of a controlled, randomized, clinical trial may not be feasible or ethically justified for all indica- tions, particularly in life-threatening diseases, where there is no satisfactory standard of care. For example, approval for a trial that requires surgery to administer the advanced product is quite challenging and requires strong justification in order to be approved.

For this reason, such innovative therapies with a number of uncertainties discourage physicians from recommending them to their patients, because the outcome of the patient's administration and follow-up will initially burden the treating physician [7].

The clinical success of these products depends on the ability of clinicians and their teams to perform such treatments. A prerequisite is the specialized training of health care providers to ensure product quality through standard operating procedures (SOPs), from the collection of the product and treatment process to extensive patient follow-up after administration.

Traceability requirements for advanced therapy products are the same as those for medical products of human origin, medical products of human origin (MPHO) (e.g., organs, tissues, cells, etc.), but patient's file should be stored longer (over

Table 2. ATMPs Borderline Source: Alliance for Regenerative Medicine 2019

\begin{tabular}{ll}
\hline & Advance therapy medicinal products borderlines \\
\hline Advantages & Disadvantages \\
\hline Highly personalized therapy & Complex manufacturing \\
Direct application through infusion & High up-front cost \\
Longer lasting effect & One-time treatment \\
Address complex diseases & Specific regulatory and pharmacovigilance demands \\
Improving health-related quality of life & Highly specific storage requirements: short shelf life \\
Fewer hospitalizations, comorbidities and associate treatment & \\
\hline
\end{tabular}

ATMPs: advanced therapy medicinal products. 
30 years as defined for MPHO). Also, in the case of cellular products, extensive documentation is required to provide the donated biological material. This is particularly important in the case of autologous transplants, where the procedure should be documented and traced at all stages in order to ensure that the biological material is returned to patients.

Traceability also covers the ability to identify and track all relevant data concerning products and materials related to advanced therapy products, as well as confirmation that their application has actually taken place. The same policy should be followed to very sensitive products such as the ones with short shelf life or products requiring special transport conditions.

Apart from the challenges faced by these products at systemic level, the introduction of advanced therapy products into clinical practice is often accompanied by practical issues such as the patient's days of stay in the clinic and/or the requisite specialized staff. This has as a result that the clinic's management is reluctant to direct the management of these products [8]. Additionally, regarding staff selection, clinics that use advanced therapy products are often in the dilemma of selecting experienced staff not only at the clinical level but also at the institutional level due to the limited use of these products into the daily operation of the clinic.

\section{The European Experience: The Creation of the Regulatory Framework}

In the absence of a clear legal framework, the first attempt to exploit ATMPs was carried out by the same bodies that developed them. The research laboratories, in the context of an applied service, received cells or tissues from the patient himself or from a relative donor (at the request of the treating physician) and after processing them, were sent to the treating physician in order to be administered to the patients. These were personalized treatments, prepared for a particular patient. Then an attempt was made to systematize ATMP production from academic research centers through spin-off companies and biotech small and medium-sized enterprises.

Therefore, the European Commission (EC) took initiatives in order to set standards and operating rules. After extensive consultation, the European Union (EU) Regulation 1394/2007 [9] was published on advanced therapy medicinal products and for amending Directive 2001/83 (community code for medicinal products for human use) and Regulation 726/2004 (community code for the European Medicines Agency (EMA)).

The new regulation sets specific rules on the authorization and supervision of advanced therapy medicinal products and on pharmacovigilance in relation to them. The responsible central body for the licensing of the ATMPs is the EMA and the local competent bodies of the national drug organizations of the member states. At the same time, the Committee for Advanced Therapies (CAT) was established, the main role of which is to advise on the classification of a product [6].

CAT decides whether a product is an ATMP and then registers it in the related category (gene therapy drug, somatic therapy, tissue engineered product or combination of categories). Furthermore, since the classification of these products often depend on technical details; CAT makes scientific recommendations as to whether the products fall within the definition of advanced therapies. However, member states do not have to abide by these recommendations at EU level, therefore, each state applies a different legal framework for similar products. Laboratories developing ATMPs often complain about these classifications, where they often have to stop applying standard therapy protocols. It is therefore clear that obtaining a license from EMA, to produce ATMPs was a prerequisite for the export of ATMPs from one member state to another and for their off-the-shelf sale.

\section{Regulatory Exceptions: "Hospital Exemption" Framework}

In addition, the Regulation (EU) 1394/2007 left open the possibility of exceptions. In particular, the "hospital exemption" [10] allows for the administration of an ATMP without a license, provided that all the following conditions are met: 1) Their production is not systematic; 2) High quality standards are followed; 3) Prepared and used within the same member state; 4) They are administered in a hospital; 5) It is administered under the responsibility of the attending physician; 6) They are manufactured after a medical order and for a particular patient.

National medicines agencies are responsible for the application of hospital exemptions and authorization. However, in accordance with Article 5 (1) of Directive 2001/83 / EC, it appears the possibility of being opt-out from EMA authorization. This Article states that "A member state may, in accordance with the legislation in force and in order to meet special needs, be excluded from the application of the provisions of this directive medicinal products supplied on a bona fide, prepared in accordance with the specifications of an approved medical practitioner and intended to be administered to his patients under his direct personal responsibility." Adaptation to the EU legislation was granted until 2012.

\section{The Field of Advanced Therapy Products Today}

Although the Regulation 1394/2007 has led to the commercial exploitation of ATMP and facilitated the entry of large pharmaceutical companies into the field, the reality today, 11 years after its first implementation, is completely different. While CAT has already registered 285 products as ATMPs, out of the total of 313 that were called to register, only 10 licenses were granted in the same period, of which only six remained (the rest related to products withdrawn).

On the contrary, many research laboratories and companies are continuing their activities, as before the implementation of community legislation, under the umbrella of the hospital exemption. Countries that have been fully harmonized with the Regulation EU/1394/2007 are seeking approval by the national competent body. Thus, in Germany nine "products" have national permits, eight as well as in Poland, the UK 18 ATMP are in production under the Article 5 (1) of Directive 2001/83/EC; 
while for other countries there is no information available [11]. At the same time more than 900 clinical trials involving ATMP are in progress, most of them in phase I/II. The key players in the development and delivery of ATMP remain academic/ research centers and small and medium-sized enterprises $[8$, 12], while the involvement of pharmaceutical companies is focusing on recent developments in the treatment of oncological incidents with in vitro modified cytotoxic $\mathrm{T}$ lymphocytes and chimeric antigen receptor (CAR)-T cells [13].

\section{Managing and Assuring the Quality of ATMPs}

Safe and sustainable disposal of processed tissues and cells is a key pillar of modern EU health care and is a priority for both national and health authorities. While tissues and cells are usually donated without any remuneration, further processing and handling is required before being applied as an advanced treatment to the recipient [14].

The establishment of such a laboratory for the production of advanced therapy products requires equipment, know-how and appropriate facilities. A prerequisite is the construction of clean rooms [15] that offer environmental conditions for the safe processing of tissues and cells. Rapid advances in ATMP have challenges among a variety of legal uncertainties that make it hard for researchers and businesses to understand the quality and safety requirements as defined by the EC.

In particular, the EC, in order to assist member states in dealing with critical processes arising mainly from the understanding and implementation of the legislative framework, has developed guidelines in order to bring together various documents, and in particular various pieces of EU legislation, into a single document, processes regarding the proper operation of Good Manufacturing Practices (GMPs). These guidelines govern all stages of the chain from donation to application (donation, procurement, testing, coding, processing, distribution, and application) of processed products derived from human tissues and cells.

Compliance with GMP standards has been a major challenge for the progress of ATMP in Europe. In fact, these difficulties are a primary reason why, till 2014, only four drugs, under the ATMP regulation, were authorized through the centralized EU drug licensing process [16]. For this reason, the EC has developed a 2-year program under FP7 with the acronym AGORA [17] (ATMP GMP Open Access Research Alliance) coordinated by the UK and designed to create a source of information to support biomedical and clinical research through a platform. This platform facilitates the consultation among researchers working in the field, as well as support for ATMPs academic programmers to comply with legislation, while promoting the development of early-stage therapies to commercial trials.

The results of this project, whose participants were mainly academics from the UK, German and Dutch Universities, led to the creation of new knowledge about the impact of advanced therapy regulations between member states on: 1) The comparative use of the experience of the partners and the invited parties of the project; 2) Conducting a European survey of non-industrial establishments in this field; 3 ) The organization of workshops, major conferences and joint meetings with European Society for Blood and Marrow Transplantation (EBMT) for the development of a targeted and collaborative dialogue; 4) Analysis of publications and guidelines for better future legislation; 5) Analysis of innovation statistics in relation to advanced therapies.

An important problem in the field of advanced therapies is to assess the quality of raw material, which often might include high-risk raw materials. The availability of these therapies in very high quality was initially limited because they were mainly sold "for research use only" and were accompanied by limited product documentation by the suppliers [18]. With the accumulation of experience and as the use of these products has been transferred to humans, the operating costs of the laboratories that produce ATMPs have been increased and distributed among the four main activities required for tissue/ cell transformation from a donor to a treatment for a recipient: 1) Donor supply: donor location, informed consent, donor eligibility, tissue/cell removal; 2) Control: virological and bacteriological testing; 3 ) Treatment and storage: the transformation of tissues/cells into a product ready for application as a treatment to the recipient; 4) Distribution: for sending the final product to the clinician or hospital where the tissue/cell will be administered to a patient.

\section{Bio-Vigilance System and Quality Standards}

The above activities, which involve the use of products of human origin, from donation to follow-up, are exposed to the risks of specific complications that are mainly related to the transmission of infectious diseases and other adverse effects. For this reason, the bio-vigilance system is a strategic tool for ensuring the quality and safety of tissues and cells, intended for the application of advanced therapies to humans. The biovigilance system shall focus on the prevention of errors and along with the strong involvement of clinicians will maintain a consistent standard of agreed assays for treated tissues and cells intended for clinical application, therefore, being an important factor in facilitating innovation [19].

This data will allow competent authorities to monitor and ensure the quality, safety and functionality of new treatments. The Committee for Advanced Therapies has expressed the need for the development of specific quality standards for each cell therapy. However, as ATMPs are at the forefront of scientific discovery, quality standards have to be updated very quickly. However, there is a risk, that by the time the standards are implemented, these products might be overcome by a new technology.

Finally, the future development of the ATMP sector also depends on state-of-the-art technologies, particularly in the field of quality control. However, some of these new technologies may be very expensive, especially for EU member states with lower gross domestic product (GDP) rates. Therefore, the added value of these technologies needs to be reassessed at national level so that healthcare policy can respond to the safety and the quality of the ATMPs [20]. 
The new bio-vigilance system should develop as a learning organization and shall be based on the human resources system, which shall be characterized by continuous staff training, communication of health professionals between different disciplines, as well as the establishment of an organizational culture of quality and safety for the patient and staff.

\section{Acknowledgments}

This article was supported by the Postgraduate Health and Social Care Management Programme of the University of West Attica, the Center of Clinical Research, Experimental Surgery and Translational Research and Hellenic Cord Blood Bank of Biomedical Research Foundation Academy of Athens. We thank our colleagues who provided insight and expertise that greatly assisted this article.

\section{Financial Disclosure}

None to declare.

\section{Conflict of Interest}

MN has received grants by the Deutsche Forschungsgemeinschaft (DFG) through the Sonderforschungsbereich Transregio 19 "Inflammatory Cardiomyopathy" (SFB TR19) (TP B2), and by the University Hospital Giessen and Marburg Foundation Grant "T cell functionality" (UKGM 10/2009). MN has been consultant to the IKDT (Institute for Cardiac Diagnosis and Therapy GmbH, Berlin) 2004 - 2008, and has received honoraria for presentations and/or participated in advisory boards from Abiomed, AstraZeneca, Bayer, Boehringer Ingelheim, Bristol-Myers Squibb, Fresenius, Miltenyi Biotech, Novartis, Pfizer and Zoll. All other authors declare no potential conflict of interest.

\section{Author Contribution}

A.G. conceived of the presented idea, V.G. wrote the manuscript with support from M.K. and E.F.S., M.N. contribute to the final version of the manuscript, E.M. and C.S helped supervised the manuscript and A.K. supervised the project.

\section{Data Availability}

The authors declare that data supporting the findings of this study are available within the article.

\section{Abbreviations}

ATMPs: advanced therapy medicinal products; EC: European Commission; EU: European Union; GTMP: gene therapy me- dicinal product; CBMP: cell based medicinal products; IPS: induced pluripotent stem cell; MPHO: medical products of human origin; CAT: Committee for Advanced Therapies; EMA: European Medicines Agency

\section{References}

1. Sotiris Soulis, Markos Sarris, George Pierrakos, Aspasia Goula, George Koutitsas, Vassiliki Gkioka. Public health policies in European Union: an innovation strategy - Horizon. 2020. In: Catherine Stavropoulos-Giokas, Dominique Charron, Cristina Navarrete. Cord blood stem cells and regenerative medicine. Elsevier; 2015:347-363.

2. Directive 2001/83/EC of the European Parliament and of the Council of 6 November 2001 on the community code relating to medicinal products for human use. Official Journal L 311. 2001:0067-0128.

3. Directive 2004/23/EC of the European parliament and of the Council of 31 March 2004. L102. 2004. p. 48.

4. MacNeil S. Progress and opportunities for tissue-engineered skin. Nature. 2007;445(7130):874-880.

5. Giulia Detela, Anthony Lodge. EU regulatory pathways for ATMPs: standard, accelerated and adaptive pathwaysto marketing authorisation; Molecular therapy: methods and clinical development. 2019;13.

6. The Committee for Advanced Therapies (CAT) and the CAT Scientific Secretariat; Challenges with advanced therapy medicinal products and how to meet them; Nature Reviews Drug Discovery. March 2010.

7. Abou-El-Enein M, Elsanhoury A, Reinke P. Overcoming challenges facing advanced therapies in the EU market. Cell Stem Cell. 2016;19(3):293-297.

8. Ten Ham RMT, Hoekman J, Hovels AM, Broekmans AW, Leufkens HGM, Klungel OH. Challenges in advanced therapy medicinal product development: a survey among companies in Europe. Mol Ther Methods Clin Dev. 2018;11:121-130.

9. Regulation (EC) no 1394/2007 of the European Parliament and of the Council of 13 November 2007 on advanced therapy medicinal products and amending Directive 2001/83/EC and Regulation (EC) No 726/2004 Official Journal of the European Union L324/121.

10. Ivaskiene $\mathrm{T}$, Mauricas M, Ivaska J. Hospital exemption for advanced therapy medicinal products: issue in application in the European Union Member States. Curr Stem Cell Res Ther. 2017;12(1):45-51.

11. Elsanhoury A, Sanzenbacher R, Reinke P, Abou-El-Enein $\mathrm{M}$. Accelerating patients' access to advanced therapies in the EU. Mol Ther Methods Clin Dev. 2017;7:15-19.

12. Hanna E, Remuzat C, Auquier P, Toumi M. Advanced therapy medicinal products: current and future perspectives. J Mark Access Health Policy. 2016;4:31036.

13. Fesnak AD, June CH, Levine BL. Engineered T cells: the promise and challenges of cancer immunotherapy. Nat Rev Cancer. 2016;16(9):566-581.

14. Economic landscapes of human tissues and cells for clinical application-EAHC/2012/Health/19 Contractno 20126301. 
15. EudraLex - Volume 4 - Good Manufacturing Practice (GMP) guidelines.

16. Milmo S. GMP challenges for advanced therapy medicinal products. BioPharm International. 2015;27(12).

17. AGORA Final Report; Dec 2015; Grant agreement ID: 602366.

18. Salmikangas P. Advanced therapies and the RCG working party - preliminary aspects. Presentation at workshop on 50 years of EDQM Leadership in the Quality of Medicines (Strasbourg, October 2014).

19. Gkioka V, Sarris M. Quality management and assurance: implementation guide and benefits for transplant coordination services. In: Alkiviadis Costakis, Markos Sarris. Transplantation Systems - Best Practices of Quality and Safety. Broken Hill Publishers LTD, 2016.

20. Annual Review 2017/18; Human tissue authority. 